\title{
ULTRASONOGRAPHIC DIAGNOSIS OF PNEUMONIA IN CHILDHOOD: A LITERATURE REVIEW
}

\author{
MARIANA BAMONTE SEOANE, JOSÉ HÉRACLES RODRIGUES RIBEIRO DE ALMEIDA, LÍVIA FRANCHI, LEONARDO DE SOUZA PIBER
}

\begin{abstract}
OBJECTIVES: Review the ultrasound technique and establish the diagnostic benefits brought in relation to primary investigation methods for pneumonia in pediatrics.

METHODS: This is a narrative literature review with search in the main databases: PubMed, Cochrane Library and Scielo, using the MeSH terms in the search strategy: Pneumonia AND Child AND Ultrasonography. As inclusion criteria, we used randomized clinical trials, systematic reviews and narratives of the literature, as well as case reports on the subject. As an exclusion criterion, articles whose theme touched the central theme or which presented the theme SARS-CoV-2 were removed through manual review by two authors.

RESULTS: Pneumonia is one of the three leading causes of death in pediatric settings worldwide. The lack of an exam considered the gold standard, capable of diagnosing pneumonia with a high degree of accuracy, drives the search for new diagnostic methods. The increasing use of PointOf-Care (POCUS) ultrasound in emergency rooms is sparking interest in considering it a first-line test. The non-exposure of pediatric patients to radiation, the reduction in cost compared to chest $X$-rays, in addition to the high sensitivity (96\%) and specificity (93\%), corroborate the perspective that the method is increasingly closer to being implanted in the gold standard diagnosis of the investigation, although, to define it as such, it is necessary to analyze other broader variables.

CONCLUSION: Despite the benefits, further studies are needed in order to elucidate the capacitive potentials of the POCUS, thus promoting the implementation of an adequate diagnostic tool.
\end{abstract}

\section{KEYWORDS: PNEUMONIA, ULTRASONOGRAPHY, RADIOLOGY, CHILD, DIAGNOSTIC IMAGING.}

\section{INTRODUCTION}

Lower respiratory tract infections are pathologies with a significant impact in terms of morbidity, mortality and hospitalization, whether in the adult population or in the pediatric age group. In children, the problem takes on even greater proportions, mainly referring specifically to a clinical condition: pneumonia. ${ }^{1}$

Pneumonia is one of the three leading causes of death in the pediatric context in the world, accounting for a significant epidemiology with about one million deaths per year in children under five years of age. Another major problem of this pathology is the significant impact on public health expenses, especially in developing countries, since many cases evolve to prolonged hospitalization and may even require admission to an intensive care unit. ${ }^{2-4}$

On the other hand, some aspects related to the improvement of living conditions, especially adequate nutrition, preventive aspects and good vaccinal adherence of the main pathogens of this pathology, show us a downward transition in relation to its incidence. ${ }^{4}$

Although the incidence of this pathology in the pediatric age group is declining, there are still problems related to diagnosis. Currently, there is still no exam considered the gold standard capable of diagnosing pneumonia with a high degree of accuracy. ${ }^{3,4}$

The clinical findings of pneumonia consist of symptoms commonly present, also, in viral respiratory infections such as fever, cough and tachypnea, reflecting a great conflict between possible differential diagnoses. This fact, together with the non-specific findings of the physical examination and the lack of an imaging exam with reliable specificity and sensitivity, reflect the difficult specific diagnosis of pneumonia, a pathology in which an early diagnosis prevents future complications. 3,4

The test considered as the first line to start the investigation of a suspected condition of pneumonia is the chest $\mathrm{X}$-ray, however, it cannot be considered the gold standard since it has low sensitivity and specificity, is a dependent examiner and exposes pediatric patients to radiation. ${ }^{5-9}$

Given this scenario and the new perspectives with the increasing availability and use of ultrasonographic methods, the benefits of this modality for diagnosing pneumonia in the pediatric setting have become notorious, exemplifying the non-exposure to radiation, less economic impact. There-
1.Universidade Santo Amaro
MAILING ADDRESS

LEONARDO DE SOUZA PIBER

Endereço: Rua Marechal Deodoro, 135 apto 62B, Santo Amaro, São

Paulo, SP, Brasil,

CEP 04738-000

E-mail: leonardopiber@gmail.com 
fore, this study aims to bring what is most current and relevant about the topic that can directly affect the paradigms of current medical practice, in the case of the ultrasound diagnosis of pneumonia in childhood.

\section{OBJECTIVE}

The study aims to review the ultrasound technique as well as to establish the diagnostic benefits brought in relation to primary investigation methods for pneumonia in pediatrics.

\section{MATERIALS AND METHODS}

The present study consists of a narrative review of the literature. To identify the articles on the subject, on July 27, 2021, a search was carried out in the main databases: PubMed, Cochrane Library and Scielo, using descriptors in English, Spanish and Portuguese, according to the requirements of the database in the search strategy: Pneumonia AND Child AND Ultrasonography. This search obtained the results described in table 1 :

\begin{tabular}{|l|l|}
\hline DATA BASE & RESULTS \\
\hline PUBMED & 216 \\
\hline COCHRANE & 5 \\
\hline SCIELO & 2 \\
\hline
\end{tabular}

Table 1: Search results for articles in their entirety, without inclusion criteria.

After the first search for inclusion of the screened articles, the following criteria were used: publications in the three predetermined languages, from the last five years (20162021), resulting in a total of 132 articles, 129 on the PubMed platform and one on the platform Cochrane Library and two on the Scielo platform.

As a means to establish the best in the literature, the following types of articles were also selected as inclusion criteria after the first search: clinical trial, randomized clinical trial, systematic review and meta-analysis, narrative literature review and report of cases. Resulting in a total of 40 articles (PubMED), one article (Cochrane) and two articles (Scielo).

In order to be as homogeneous as possible, articles (18) containing the use of ultrasonography in patients diagnosed with SARS-CoV-2 (18) (coronavirus) were excluded.
For the purpose of avoiding bias and duplicate articles, two reviewers performed the analysis based on the abstracts of publications, excluding duplicate articles in the databases and articles whose abstracts did not present the approach to the proposed theme.

After applying all criteria, a total of 17 articles were selected, which were read in their entirety by a group of two reviewers, who wrote the present study.

\section{RESULTS AND DISCUSSION}

Ultrasonography, still little used as a diagnostic method, is usually performed as a complementary exam to radiography in more complicated cases. Currently, with the growing search for new methods and the increasingly recurrent use of this tool in emergency and emergency rooms using the Point-Of-Care ultrasound method (POCUS), there is an interest in considering it as a first-rate exam line for diagnosing pneumonia. According to the World Health Organization, $3 / 4$ of the world population does not have access to modern imaging equipment, making the "point of care" ultrasound a very important diagnostic tool. ${ }^{2,3}$

The technique consists of a systematic analysis of the chest, in the transverse, parasagittal and coronal planes, with assessment of the anterior and posterior midclavicular line and in the mid-axillary region up to the diaphragm (Figure 1), with the choice of a linear transducer that allows better visualization. . $^{1.10}$

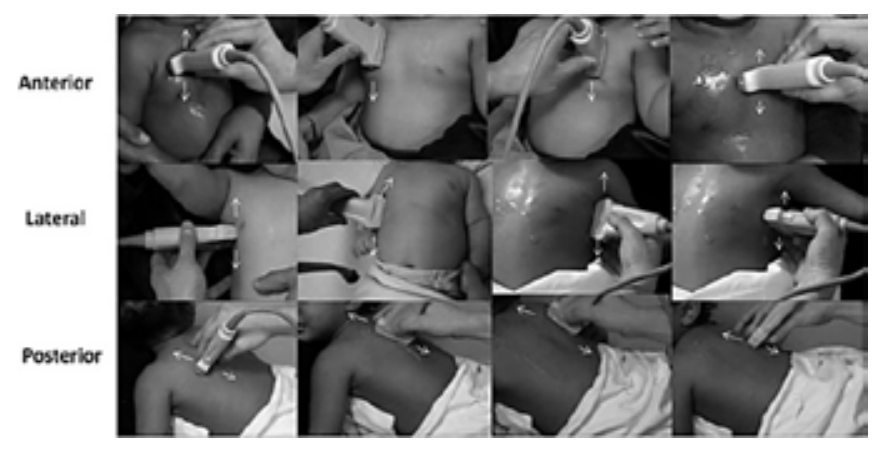

FIGURE 1: Systematic approach for evaluating the entire chest during the procedure. Adapted from: Vazquez et al, $2016^{10}$.

Ultrasonographic findings consist of understanding the pathophysiology of the entire process of lung disease, taking into account the air-fluid ratio present in the parenchyma. A healthy lung is well aerated and, therefore, it is not possible to visualize its functional structure, but only the images generated by the transducer itself. The presence of air in the lung parenchyma acts as a deflector of the waves emitted by the device, so that when the waves reflect on the pleuropulmonary interface, they form a mirror image. ${ }^{11}$

The lung parenchyma, when affected by some pathology, loses areas that were previously aerated, being re- 
placed by regions that will have a predominance of fluid. When there is total loss of aeration, it is possible to visualize the parenchyma. ${ }^{11}$

On ultrasound, there is a representation of two lines, the A-lines, horizontal, representing a normal aerated lung, and possibly the B-lines, vertical, always being pathological (Figure 2). 10.11

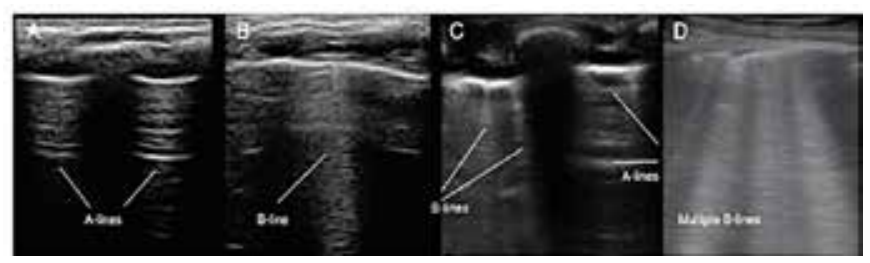

FIGURE 2: A-lines - hyperechogenic, horizontal and parallel (A); B-lines hyperechogenic and transverse (B); Lines $A$ and $B$, perpendicular to each other (C); Multiple B-lines (D). Retrieved from: Toro et al $2021^{11}$

The A-lines are formed by the reflection of waves emitted by ultrasound at the pleuropulmonary interface, they are hyperechoic, horizontal, parallel and equidistant from each other. They indicate that the lung is well aerated and may even represent a pneumothorax. The pleura will also have its image reflected in the form of a horizontal and hyperechoic line, however, it will be possible to visualize its movement."

The B-lines are hyperechoic and perpendicular to the A-lines, promoting their erasure. The presence of this line is also related to the air/fluid ratio present in the lung, being present in greater numbers when there is lung parenchyma affection. ${ }^{11}$

Consolidation, in turn, very characteristic although not pathognomonic of pneumonia, is represented on ultrasound by hypoechogenic images, with ill-defined borders and presence of vertical artifacts in adjacent areas, formed by the projection of the lung parenchyma, when there is at least involvement of two lung segments ${ }^{11,13}$ (Figures 3, 4 and 5).

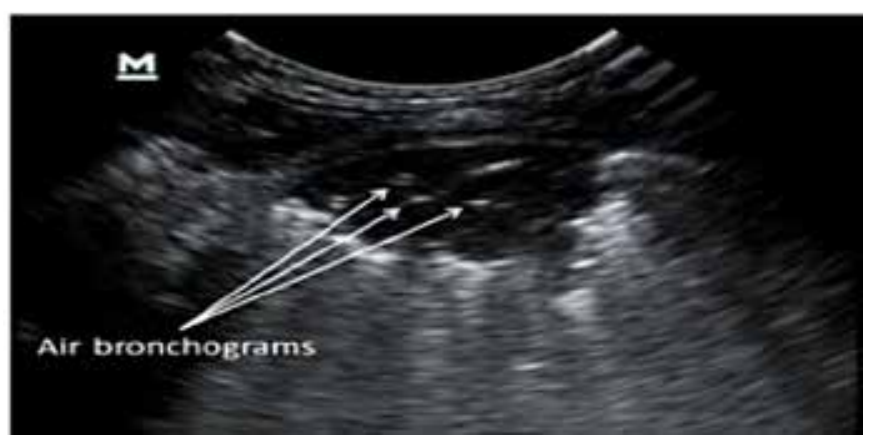

FIGURE 3: Consolidation with air bronchogram. Retrieved from: Stadler et al $2017^{2}$

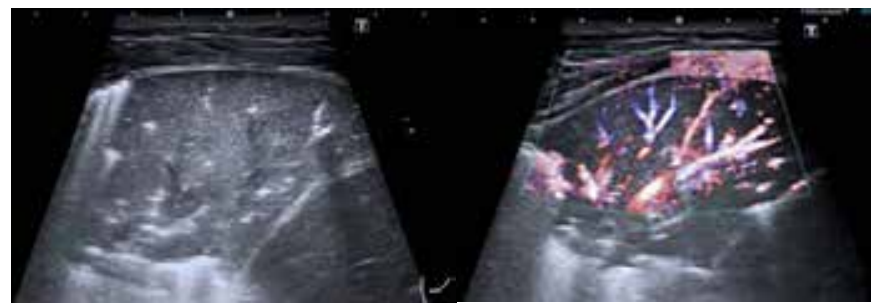

FIGURE 4: Consolidation with dynamic air bronchogram, without and with Doppler analysis.Taken from: Najgrodzka et al, $2019^{1}$.

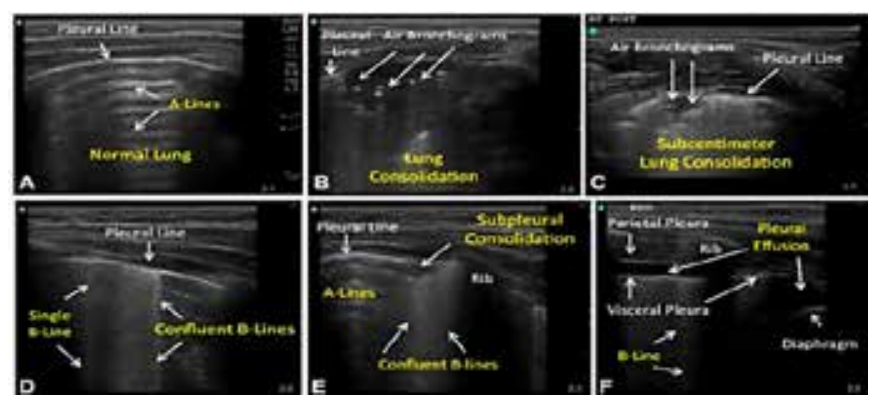

FIGURE 5: Ultrasound images of the lung. Normal lung with the presence of A-lines (A); Focal, radiographically apparent pneumonia - greater than $1 \mathrm{~cm}$ - of pulmonary consolidation with air bronchograms (B). Focal, radiographically occult pneumonia - pulmonary consolidation $<1 \mathrm{~cm}$ with air bronchograms (C). B-lines, confluent B-lines, subpleural consolidation most commonly associated with viral pneumonia or bronchiolitis; no visible ultrasonographic air bronchograms (D-E). Pleural effusion - anechoic space - between the lung and the chest wall or diaphragm (F). Retrieved from: Jones et al, $2016^{3}$.

The ultrasound image formed by the consolidation of pneumonia is very similar to that of atelectasis, and the figure often raises diagnostic doubt. It is then possible to differentiate the image of pneumonia from atelectasis by observing respiratory variance in air bronchograms, which are branched echogenic structures present in the consolidated area, commonly present in pneumonia. ${ }^{12}$

In the pediatric context, the thymus is an important organ to be differentiated in imaging exams, especially in cases of suspected pneumonia. The thymus is a more prominent organ in young children, located in the parasternal region, being represented by a hyperechoic image known as "starry sky", with no air bronchogram (Figure 6). The image of the thymus is usually adjacent to the consolidation and, therefore, its distinction on ultrasound becomes increasingly relevant. In these cases, chest radiography can also be associated with ultrasound to corroborate the diagnosis. ${ }^{13}$ 


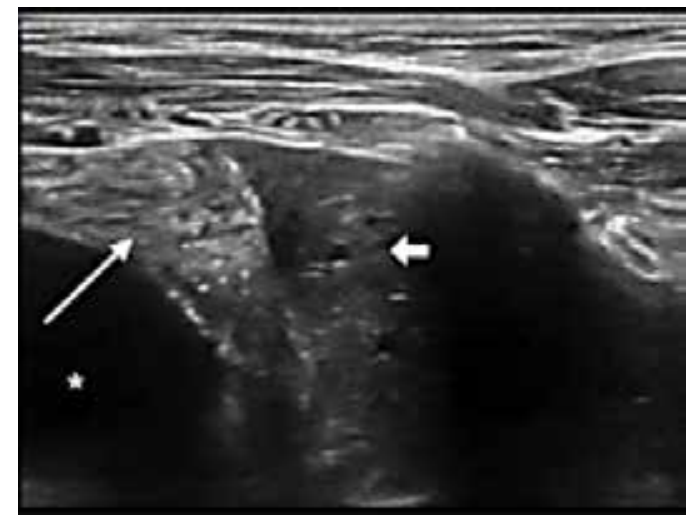

FIGURE 6: Thymus represented by the larger white arrow on the left, with the characteristic of "starry sky", while the smaller arrow on the right represents consolidation with hepatization and fluid bronchograms compatible with a picture of pneumonia. Retrieved: Harvey et al, $2017^{13}$

Chest radiography is currently still the exam of choice for diagnosing pneumonia. With the rise in the use of Pointof-Care ultrasound associated with the lack of availability of the radiography device in various hospital settings, as well as the presence of international evidence-based studies demonstrating the accuracy of POCUS in various contexts for the diagnosis of pneumonia, made the scientific community wonder about making it the exam of choice for pediatric diagnosis of this pathology. ${ }^{3}$

In recent years, several studies have evaluated the reliability of ultrasound as a diagnostic method for pneumonia in the pediatric setting and most of the results obtained were favorable, since the sensitivity, specificity, positive and negative predictive values were similar or in some cases even better than chest X-ray. A recent meta-analysis, aimed at evaluating the performance of ultrasonography, resulted in a sensitivity of $96 \%$ and a specificity of $93 \%$, with the main findings being: consolidation, positive air bronchogram, abnormal pleural lines and pleural effusion. ${ }^{12,14,15}$

In most of the literature that presented disagreement regarding the result, the chest X-ray was not able to identify some cases precisely observed by ultrasonography. This fact occurred mainly in radiographs where only the anteroposterior and/or posteroanterior projections were analyzed, in which the consolidation was seen in regions that the radiography does not accurately assess. In addition, the accuracy of radiography was much lower when it was necessary to differentiate between alveolar and interstitial pneumonia. ${ }^{4,12}$

The American Academy of Pediatrics recommends caution regarding the use of chest radiography, mainly because of the exposure of very young patients to radiation, besides disagreement in the interpretation of the result, delay in the delivery of the image as well as the inability to repeat the exam. ${ }^{1}$

Ultrasonography also stands out in the scenario of non-ex- posure to radiation, in relation to monitoring and follow-up, especially regarding the response to antibiotic therapy. The evaluation of the response obtained by performing the USG 48 hours after antibiotic therapy can be beneficial by decreasing the exposure to radiation from a new radiograph, for example, and showing classic signs of re-aeration of the union itself or of the bronchogram formed16 (Figure 7).

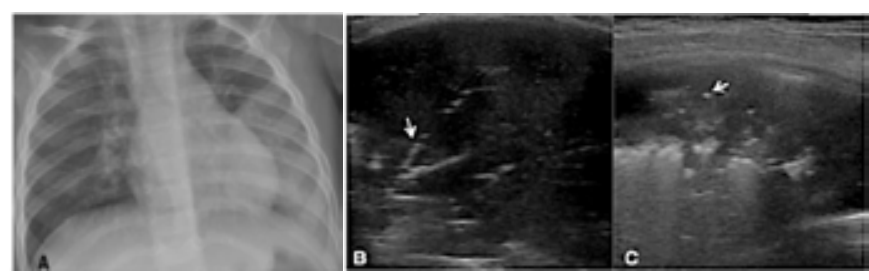

FIGURE 7: Initial assessment performed using a chest X-ray of a patient with pneumonia on the left (A); Ultrasonography demonstrating a large area of hepatization and consolidation of the lung parenchyma (B); UItrasonography after $\mathbf{4 8}$ hours of treatment demonstrating consolidation of the consolidation process, as well as more visible air bronchograms (C). Retrieved from: Buonsenso et al, $2020^{16}$.

Although most studies to date are in favor of ultrasound with expressive numbers of sensitivity and specificity of the test, the choice as a substitute method or even as a screening depends on multiple variables not obtained in the current literature. Clinical aspects that are relevant from a practical point of view, such as what should be done from a negative initial ultrasound, the request for additional evaluation with other diagnostic methods, or issues involving the safety of not prescribing antibiotic therapy when the initial assessment via ultrasound is normal, encourage further discussions that a priori revoke the establishment of the method as the gold standard in the initial screening. ${ }^{2,3}$

A recently published article evaluated the diagnostic accuracy of ultrasonography when performed by experienced physicians versus newly trained ultrasonographers. During the study, newly graduated physicians in the specialty received, for a week, practical training and didactic classes on ultrasound and even so, their results were insufficient when compared to more experienced professionals. ${ }^{17}$ This need for specific training to obtain results expressives is very well described in the literature and demonstrates the need for specific training or early and continuous contact, both in undergraduate and in specialization, either with the POCUS methodology or with conventional ultrasonography itself to obtain accurate diagnoses..$^{11,17}$

\section{CONCLUSION}

Amidst all this scenario under the presence of two main parallels, namely, the high rate of morbidity and mortality of the disease and the rise of an increasingly accessible method from which a less invasive perspective and results similar to the current diagnostic method, more and more studies are 
needed in order to elucidate its capacitive potentials to increasingly implement this early diagnostic tool in the context of pneumonia in the pediatric age group.

\section{REFERENCES}

1) Najgrodzka, P., Buda, N., Zamojska, A., Marciniewicz, E., Lewandowicz-Uszynska, A. Lung ultrasonography in the diagnosis of pneumonia in children-a metaanalysis and a review of pediatric lung imaging. Ultrasound Quarterly, 2019;35(2): 157-163.

2) Stadler, J. A., Andronikou, S., Zar, H. J. Lung ultrasound for the diagnosis of community-acquired pneumonia in children. Pediatric Radiology, 2017;47(11): 1412-1419.

3) Jones, B. P., Tay, E. T., Elikashvili, I., Sanders, J. E., Paul, A. Z., Nelson, B. P., Tsung, J. W. Feasibility and safety of substituting lung ultrasonography for chest radiography when diagnosing pneumonia in children: a randomized controlled trial. Chest, 2016;150(1): 131-138.

4) Orso, D., Ban, A., Guglielmo, N. Lung ultrasound in diagnosing pneumonia in childhood: a systematic review and meta-analysis. Journal of Ultrasound, 2018;21(3): 183-195.

5) Bada, C., Carreazo, N. Y., Chalco, J. P., Huicho, L. Inter-observer agreement in interpreting chest X-rays on children with acute lower respiratory tract infections and concurrent wheezing. Sao Paulo Medical Journal, 2007;125(3): 150-154.

6) Johnson, J. \& Kline, J. A. Intraobserver and interobserver agreement of the interpretation of pediatric chest radiographs. Emergency Radiology, 2010;17(4): 285-290.

7) Edwards, M., Lawson, Z., Morris, S., Evans, A., Harrison, S., Isaac, R., Powell, C. The presence of radiological features on chest radiographs: how well do clinicians agree? Clinical Radiology, 2012;67(7): 664-668

8) Levinsky, Y., Mimouni, F. B., Fisher, D., Ehrlichman, M. Chest radiography of acute paediatric lower respiratory infections: experience versus interobserver variation. Acta Paediatrica, 2013;102(7), e310-e314.

9) Tanaka, N., Emoto, T., Suda, H., Matsumoto, T., Matsunaga, N. Community-acquired pneumonia: a correlative study between chest radiographic and HRCT findings. Japanese Journal of Radiology, 2015;33(6), 317-328.

10) Vazquez, M., Paul, A. Z., Tay, E. T., Tsung, J. W. Evaluation and monitoring of a child with hydrocarbon pneumonitis using point-of-care lung ultrasound in the pediatric emergency department. Pediatric Emergency Care, 2016;32(9), 642-644.

11) Toro, M. S., Martínez, J. L. V., Falcão, R. V., Prata-Barbosa, A., Cunha, A. J. L. A. D. Point-of-care ultrasound by the pediatrician in the diagnosis and follow-up of community-acquired pneumonia. Jornal de Pediatria, 2021;97, 13-21.

12) Principi, N., Esposito, A., Giannitto, C., Esposito, S. Lung ultrasonography to diagnose community-acquired pneumonia in children. BMC Pulmonary Medicine, 2017; 7(1), 1-6.

13) Harvey, G., \& Tessaro, M. Child with cough and fever. Annals of Emergency Medicine, 2017;70(4), e41-e42.

14) Gravel, C. A., E Bachur, R. G. Point-of-care ultrasound differentiation of lung consolidation and normal thymus in pediatric patients: an educational case series. The Journal of Emergency Medicine, 2018;55(2), 235-239.

15) Yan, J. H., Yu, N., Wang, Y. H., Gao, Y. B., Pan, L. Lung ultrasound vs chest radiography in the diagnosis of children pneumonia: Systematic evidence. Medicine, 2020;99(50).

16) Buonsenso, D., Brancato, F., Valentini, P., Curatola, A., Supino, M., Musolino, A. M. The use of lung ultrasound to monitor the antibiotic response of community acquired pneumonia in children: a preliminary hypothesis. Journal of Ultrasound in Medicine, 2020;39(4), 817-826.

17) Tsou, P. Y., Chen, K. P., Wang, Y. H., Fishe, J., Gillon, J., Lee, C. C., Yu, D. T. Y. Diagnostic Accuracy of Lung Ultrasound Performed by Novice Versus Advanced Sonographers for Pneumonia in Children: A Systematic Review and Metaanalysis. Academic Emergency Medicine, 2019;26(9), 1074-1088. 